\title{
Lack of efflux mediated quinolone resistance in Salmonella enterica serovars Typhi and Paratyphi A
}

\author{
Sylvie Baucheron ${ }^{1,2 *}$, Isabelle Monchaux ${ }^{1,2}$, Simon Le Hello ${ }^{3}$, François-Xavier Weill ${ }^{3}$ and \\ Axel Cloeckaert ${ }^{1,2 *}$ \\ 1 UMR1282 Infectiologie et Santé Publique, Institut National de la Recherche Agronomique, Nouzilly, France \\ 2 UMR1282 Infectiologie et Santé Publique, Université François Rabelais de Tours, Tours, France \\ ${ }^{3}$ Institut Pasteur, Unité des Bactéries Pathogènes Entériques, Centre National de Référence des Escherichia coli, Shigella et Salmonella, Paris, France
}

\section{Edited by:}

Kunihiko Nishino, Osaka University,

Japan

\section{Reviewed by:}

Kunihiko Nishino, Osaka University,

Japan

Peter Heisig, University of

Hamburg, Germany

\section{*Correspondence}

Sylvie Baucheron and Axel

Cloeckaert, Unité Infectiologie et

Santé Publique site 213, Institut

National de la Recherche

Agronomique, 37380 Nouzilly,

France

e-mail:sylvie.baucheron@

tours.inra.fr:

axel.cloeckaert@tours.inra.fr

Salmonella enterica serovars Typhi and Paratyphi A isolates from human patients in France displaying different levels of resistance to quinolones or fluoroquinolones were studied for resistance mechanisms to these antimicrobial agents. All resistant isolates carried either single or multiple target gene mutations (i.e., in $g y r A$, gyrB, or parC) correlating with the resistance levels observed. Active efflux, through upregulation of multipartite efflux systems, has also been previously reported as contributing mechanism for other serovars. Therefore, we investigated also the occurrence of non-target gene mutations in regulatory regions affecting efflux pump expression. However, no mutation was detected in these regions in both Typhi and Paratyphi isolates of this study. Besides, no overexpression of the major efflux systems was observed for these isolates. Nevertheless, a large deletion of 2334 bp was identified in the acrS-acrE region of all S. Typhi strains but which did not affect the resistance phenotype. As being specific to $S$. Typhi, this deletion could be used for specific molecular detection purposes. In conclusion, the different levels of quinolone or $\mathrm{FQ}$ resistance in both $S$. Typhi and $S$. Paratyphi A seem to rely only on target modifications.

\section{Keywords: Salmonella, ciprofloxacin, transcriptional regulatory genes, acrS, efflux pumps}

\section{INTRODUCTION}

Enteric fever caused by the human-adapted pathogens Salmonella enterica serovars Typhi (S. Typhi) and Paratyphi A (S. Paratyphi A), B, and C, remains a major health problem (Crump and Mintz, 2010). A global epidemiologic study estimated that during the year 2000 typhoid fever caused 21.7 million illnesses and 21,7000 deaths and paratyphoid fever caused 5.4 million illnesses (Crump et al., 2004). During the past decade $S$. Paratyphi A was responsible for a growing proportion of enteric fever in Asia (Ochiai et al., 2005; Crump and Mintz, 2010). Enteric fever being associated with poor sanitation and unsafe food and water, it particularly affects children and adolescents in developing countries of Asia, Africa and Latin America (Crump et al., 2004; Bhan et al., 2005; Crump and Mintz, 2010). In developed countries, patients are most often ill-returned travellers or migrant workers (Bhan et al., 2005; Connor and Schwartz, 2005; Hassing et al., 2013).

To treat these infections, fluoroquinolones (FQ) and thirdgeneration cephalosporins have been considered as first-line drugs, owing to the resistance to ampicillin, chloramphenicol, and trimethoprim/sulfamethoxazole that appeared during the 1980s (Hassing et al., 2011, 2013). Multidrug resistance (MDR) in $S$. Typhi is encoded mainly by resistance genes carried by large conjugative plasmids and has been reported worldwide (Le et al., 2007). As a consequence of a widespread FQ usage, $S$. Typhi and $S$. Paratyphi A isolates resistant to nalidixic acid $\left(\mathrm{NAL}^{\mathrm{R}}\right.$, minimum inhibitory concentration $[\mathrm{MIC}]>16 \mathrm{mg} / \mathrm{L}$ ) and with decreased susceptibility to ciprofloxacin $\left(\mathrm{CIP}^{\mathrm{DS}}\right.$, MIC $0.125-1.0 \mathrm{mg} / \mathrm{L}$ ) have also emerged. Such $\mathrm{NAL}^{\mathrm{R}}-\mathrm{CIP}^{\mathrm{DS}} S$. Typhi and $S$. Paratyphi A have been isolated in endemic areas and also in developed countries (Roumagnac et al., 2006; Le et al., 2007; Gaborieau et al., 2010; Accou-Demartin et al., 2011; Hassing et al., 2011, 2013).

Resistance to quinolones in Salmonella spp. is mostly attributed to point mutations in the quinolone resistancedetermining regions (QRDRs) of the target genes gyrA, gyrB, parC, and parE (Cloeckaert and Chaslus-Dancla, 2001; Piddock, 2002; Velge et al., 2005; Giraud et al., 2006). For the gyrA gene, coding the A subunit of DNA gyrase, a single mutation resulting in an amino acid substitution at the position 83 (Serine to Phenylalanine or to Tyrosine) or at the position 87 (Aspartic acid to Asparagine or Glycine) has been the most frequently described in $\mathrm{NAL}^{\mathrm{R}}-\mathrm{CIP}^{\mathrm{DS}}$ S. Typhi and S. Paratyphi A isolates (Bhan et al., 2005; Renuka et al., 2005; Le et al., 2007; Gaborieau et al., 2010; Hassing et al., 2011). A second mutation leading to the amino acid change at the position 80 (Serine to Isoleucine or to Arginine) of the ParC subunit of topoisomerase IV was described to increase the CIP MIC ( $\geq 0.5 \mathrm{mg} / \mathrm{L})$ in $S$. Typhi and $S$. Paratyphi A human isolates from India (Gaind et al., 2006). Whereas three mutations, i.e., a double mutation in $g y r A$ at both codons 83 and 87 and one mutation in $\operatorname{par} C$, were shown to confer CIP resistance (MIC $>1 \mathrm{mg} / \mathrm{L}$ ) in $S$. Typhi and $S$. Paratyphi A human isolates from India or from Taiwan (Gaind et al., 2006; Lee et al., 2013).

Moreover, the varying levels of CIP resistance observed in S. Typhi and S. Paratyphi A isolates with only a single gyrA mutation suggest that other mechanisms could be involved in quinolone resistance in this serovar (Renuka et al., 2005). 
Resistance to FQ in $S$. Typhimurium has also been attributed to active efflux mechanism, due to overproduction of the AcrAB-TolC efflux system (Giraud et al., 2000, 2006; Cloeckaert and Chaslus-Dancla, 2001; Piddock, 2006). We have previously reported the contribution of the AcrAB-TolC efflux system in resistance to FQ in several MDR epidemic clones of $S$. Typhimurium, such as $S$. Typhimurium of phage types DT204 or DT104 (Baucheron et al., 2002, 2004a,b). Among the chromosomal loci affecting AcrAB-TolC expression, the ramRA locus appears to be the most important in Salmonella spp. (Abouzeed et al., 2008; Kehrenberg et al., 2009). $\operatorname{ramR}$ encodes a repressor protein (RamR) belonging to the TetR family of repressor proteins, and has been shown to be the local repressor protein of $\mathrm{ram} A$ transcription (Abouzeed et al., 2008; Baucheron et al., 2012); while $\operatorname{ram} A$ encodes a transcriptional activator protein (RamA) belonging to the AraC/XylS family of regulatory proteins (Nikaido et al., 2008; Bailey et al., 2010). The latter is involved in upregulating expression of the AcrAB-TolC system (Nikaido et al., 2008; Bailey et al., 2010). Several mutations in ramR or its binding site upstream of $\mathrm{ram} A$, affecting expression of this efflux system, have been detected in clinical isolates of serovar Typhimurium or Kentucky and of minor serovars Hadar, Infantis, Livingstone, or Schwarzengrund (Abouzeed et al., 2008; Kehrenberg et al., 2009;
Hentschke et al., 2010; Akiyama and Khan, 2012; Baucheron et al., 2013).

In the present study, we have characterized mechanisms involved in resistance to quinolones or fluroquinolones in 21 $S$. Typhi and S. Paratyphi A strains displaying different levels of resistance to these drugs and isolated from patients in France during the period 1997-2008. For a subset of strains, with suspected increased efflux activity, we investigated the occurrence of mutations in the global ram, sox and mar regulatory loci of AcrAB-TolC, and in the local acrR and acrS repressor genes of the AcrAB and AcrEF pumps, respectively (Abouzeed et al., 2008; Kehrenberg et al., 2009).

\section{MATERIALS AND METHODS BACTERIAL STRAINS}

The twenty one strains including 16 S. Typhi and 5 S. Paratyphi A selected for this study were collected by the French National Reference Center for Salmonella, Institut Pasteur, Paris, France. They were isolated in France from travellers or migrants between 1997 and 2008 (Table 1). The selection was made to obtain diversity in terms of geographic origin, year of isolation, genetic lineages (haplotype for S. Typhi; Roumagnac et al., 2006), and phenotype of resistance to quinolones (Table 1).

Table 1 | Salmonella enterica serovars Typhi and Paratyphi A strains analyzed in this study.

\begin{tabular}{|c|c|c|c|c|c|c|c|c|c|c|c|}
\hline \multirow[t]{2}{*}{ Strain } & \multirow[t]{2}{*}{ Country } & \multirow{2}{*}{$\begin{array}{c}\text { Year of } \\
\text { isolation }\end{array}$} & \multirow{2}{*}{$\begin{array}{l}\text { Haplo } \\
\text { type }\end{array}$} & \multirow{2}{*}{$\begin{array}{l}\text { Antimicrobial } \\
\text { resistance pattern }\end{array}$} & \multicolumn{2}{|c|}{$\operatorname{MIC}(\mathbf{m g} / \mathrm{L})$} & \multicolumn{4}{|c|}{ Substitution(s) in the QRDR of: } & \multirow[t]{2}{*}{ AcrA production ratio } \\
\hline & & & & & NAL & CIP & GyrA & GyrB & ParC & ParE & \\
\hline \multicolumn{12}{|c|}{ SALMONELLA TYPHI } \\
\hline $06-423$ & India & 2006 & ND & Pansusceptible & 4 & 0.015 & WT & WT & WT & WT & 1 \\
\hline $06-426$ & India & 2006 & ND & CIPDS & 16 & 0.125 & WT & S464Y & WT & WT & 1 \\
\hline $02-1180$ & India & 2002 & $\mathrm{H} 45$ & NALCIPDS & 64 & 0.125 & D87G & WT & WT & WT & 1 \\
\hline $05-3275$ & Morocco & 2005 & H6 & NALCIPDS & 64 & 0.125 & D87N & WT & WT & WT & 1 \\
\hline $4(02) \mathrm{MB}$ & Vietnam & 1997 & H58 & ASCSulTmpTeNAL & 128 & 0.03 & S83Y & WT & WT & WT & 0.5 \\
\hline 222(97)MN & Vietnam & 1996 & ND & ASCSulTmpTeNALCIPDS & 128 & 0.125 & S83F & WT & WT & WT & 0.5 \\
\hline 43(97)MN & Vietnam & 1996 & H63 & ASCSulTmpTeNALCIPDS & 128 & 0.125 & S83F & WT & WT & WT & 0.5 \\
\hline $98-3139$ & Mexico & 1998 & $\mathrm{H} 50$ & NALCIPDS & 128 & 0.125 & S83F & WT & WT & WT & 0.5 \\
\hline $02-7744$ & India & 2002 & H52 & NALCIPDS & 128 & 0.125 & S83F & WT & WT & WT & 0.5 \\
\hline 226(97)MN & Vietnam & 1996 & H61 & ASCSuITmpTeNALCIPDS & 128 & 0.25 & S83F & WT & WT & WT & 0.5 \\
\hline $97-2307$ & India & 1997 & H63 & NALCIPDS & 256 & 0.125 & S83F & WT & WT & WT & 0.5 \\
\hline 318(98)MB & Vietnam & 1998 & H58 & ASCSulTmpTeNALCIPDS & 512 & 0.25 & S83Y & WT & WT & WT & 1 \\
\hline 39(98)MN & Vietnam & 1998 & H58 & ASCSulTmpTeNALCIPDS & 512 & 0.25 & S83F & WT & WT & WT & 1 \\
\hline $4(02) \mathrm{MN}$ & Vietnam & 2000 & H58 & ASCSulTmpTeNALCIPDS & 1024 & 0.25 & S83F & WT & WT & D420N & 1 \\
\hline $5(04) \mathrm{MN}$ & Vietnam & 2004 & ND & NALCIPDS & 1024 & 0.25 & S83F & WT & WT & D420N & 1 \\
\hline 04-2176 & India & 2004 & H58 & SSpSulTmpTeNALCIP & 1024 & 8 & S83F; D87N & WT & S801 & WT & 1 \\
\hline \multicolumn{12}{|c|}{ SALMONELLA PARATYPHI A } \\
\hline 08-8903 & Senegal & 2008 & & Pansusceptible & 8 & 0.030 & WT & WT & WT & WT & 2 \\
\hline $07-6329$ & Burkina Faso & 2007 & & $\mathrm{CIP}^{\mathrm{DS}}$ & 16 & 0.25 & WT & S464F & WT & WT & 2 \\
\hline 05-208 & India & 2005 & & NALCIPDS & 256 & 0.50 & S83F & WT & WT & WT & 2 \\
\hline $08-4271$ & Guinea Bissau & 2008 & & NACIPDS & 1024 & 1 & S83F & WT & WT & WT & 2 \\
\hline 08-2580 & India & 2008 & & NALCIPDS & 1024 & 1 & S83F & WT & WT & WT & 3 \\
\hline
\end{tabular}

${ }^{*}$ AcrA expression was measured by dot blotting with an anti-AcrA polyclonal antibody.

$N D$, not determined.

WT, wild type.

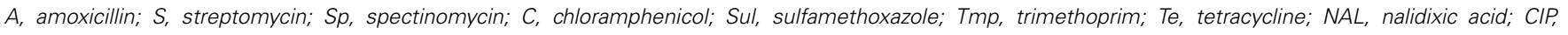
ciprofloxacin; CIPDS, decreased susceptibility to ciprofloxacin.

$D$, aspartic acid; F, phenylalanine; G, glycine; I, isoleucine; $N$, asparagine; $S$, serine; $Y$, tyrosine. 


\section{ANTIMICROBIAL SUSCEPTIBILITY TESTING}

Antimicrobial susceptibility was investigated by the standard disk diffusion method according to the recommendations of the Antibiogram Committee of the French Society for Microbiology (CA-SFM) (www.sfm-microbiologie.org/). The MICs of NAL and CIP were determined by the standard agar doubling dilution method as described previously (Baucheron et al., 2002). The $\mathrm{NAL}^{\mathrm{R}}$ isolates were defined as having a MIC $>16 \mathrm{mg} / \mathrm{L}$. The $\mathrm{CIP}^{\mathrm{R}}$ isolates were defined as having a MIC $>1 \mathrm{mg} / \mathrm{L}$ and $\mathrm{CIP}^{\mathrm{DS}}$ isolates as having a MIC comprised between 0.125 and $1.0 \mathrm{mg} / \mathrm{L}$ (Accou-Demartin et al., 2011; Hassing et al., 2013). MICs of these antibiotics were also determined in the presence of the efflux pump inhibitor Phe-Arg- $\beta$-naphthylamide (PA $\beta N$, Sigma) at the following concentrations: 10, 20, 30, 40, 50, and $60 \mathrm{mg} / \mathrm{L}$.

\section{ASSESSMENT OF TARGET-AFFECTING MECHANISMS}

Mutations in the QRDRs of $g y r A$, gyrB, parC, and parE genes were detected as described previously (Le et al., 2007; Song et al., 2010; Accou-Demartin et al., 2011).

The search of plasmid-mediated quinolone resistances genes, $q n r A, q n r B$, qnrS, qnrD, aac (6')-Ib-cr, and $q e p A$ was performed as described previously (Accou-Demartin et al., 2011).

\section{ASSESSMENT OF EFFLUX MECHANISMS}

Efflux pump production was assessed by dot blot using an antiAcrA polyclonal antibody as described previously (Abouzeed et al., 2008). Occurrence of mutations affecting $a c r A B$, acrEF, and tolC expression was determined by PCR and sequencing the regulatory regions ramR-ramA, acrR-acrA, marC-marO-marR-marA, soxS-soxR, and acrS-acrE using primers listed in (Table 2).

Table 2 | Primers used for PCRs.

\begin{tabular}{|c|c|c|c|c|c|c|}
\hline $\begin{array}{l}\text { Primer used } \\
\text { and target } \\
\text { region }\end{array}$ & Primer & $\begin{array}{l}\text { Nucleotide position } \\
\text { relative to the LT2 strain } \\
\text { genome sequence }^{*}\end{array}$ & Oligonucleotide sequence(s) $\left(5^{\prime}-3^{\prime}\right)$ & Size (bp) & $\begin{array}{l}\text { Annealing } \\
\text { temp }\left({ }^{\circ} \mathrm{C}\right)\end{array}$ & Reference \\
\hline \multicolumn{7}{|c|}{ DETECTION OF MUTATIONS } \\
\hline \multirow[t]{2}{*}{ ramR-ramA } & ram5 & 638085 & TCGGTAAAAGGCAGTTCCAG & 958 & 60 & Baucheron et al., 2013 \\
\hline & ramA6 & 639042 & GTCGATAACCTGAGCGGAAA & & & \\
\hline \multirow[t]{2}{*}{ acrR-acrA } & acrR1 & 533463 & CAGTGGTTCCGTTTTTAGTG & 992 & 58 & Olliver et al., 2005 \\
\hline & acrR2 & 534454 & ACAGAATAGCGACACAGAAA & & & \\
\hline \multirow{3}{*}{$\begin{array}{l}\text { marC-marO- } \\
\text { marR-marA }\end{array}$} & marR1 & 1597459 & CAGTGTTGCGTCTGGACATC & 787 & 60 & Baucheron et al., 2013 \\
\hline & & & & & & \\
\hline & marR2 & 1598245 & GCTAACGGGAGCAGTACGAC & & & \\
\hline \multirow[t]{2}{*}{ soxS-soxR } & sox1 & 4503970 & CTACAGGCGGTGACGGTAAT & 915 & 60 & Baucheron et al., 2013 \\
\hline & sox2 & 4504884 & CGGCGCTTTAGTTTTAGGTG & & & \\
\hline \multirow[t]{2}{*}{ acrS-acrE } & acrS3 & 3559106 & AAAACGAACGGGAACTGATG & $2874^{* * *}$ & 58 & This study \\
\hline & acrS4 & 3561978 & ACAAACATACCGGGAAGCAG & & & \\
\hline \multicolumn{7}{|l|}{ qRT-PCR } \\
\hline \multirow[t]{2}{*}{$g m k$} & gmk-f & 3933294 & TTGGCAGGGAGGCGTTT & 62 & 60 & Baucheron et al., 2012 \\
\hline & gmk-r & 3933355 & GCGCGAAGTGCCGTAGTAAT & & & \\
\hline \multirow[t]{2}{*}{$g y r B$} & gyrB-f & 4040275 & TCTССТCACAGACCAAAGATAAGCT & 81 & 60 & Baucheron et al., 2012 \\
\hline & gyrB-r & 4040195 & CGCTCAGCAGTTCGTTCATC & & & \\
\hline \multirow[t]{2}{*}{ rrs } & rrs-f & $\mathrm{NA}^{* *}$ & CCAGCAGCCGCGGTAAT & 57 & 60 & Baucheron et al., 2012 \\
\hline & rrs-r & NA** & TTTACGCCCAGTAATTCCGATT & & & \\
\hline \multirow[t]{2}{*}{$\operatorname{ram} A$} & $\operatorname{ram} A-f$ & 639180 & GCGTGAACGGAAGCTAAAAC & 167 & 60 & Baucheron et al., 2012 \\
\hline & ramA-r & 639346 & GGCCATGCTTTTCTTTACGA & & & \\
\hline \multirow[t]{2}{*}{$\operatorname{ram} R$} & ramR-f & 638623 & TAACGCAGGTGTTGCAGAAG & 192 & 64 & Baucheron et al., 2012 \\
\hline & ramR-r & 638432 & TGGTTCAGACCCCAACTGAT & & & \\
\hline \multirow[t]{2}{*}{ acrA } & acrA-f & 533120 & GAAACCGCACGTATCAACCT & 220 & 60 & Baucheron et al., 2012 \\
\hline & acrA-r & 532901 & CCTGTTTCAGCGAACCATTT & & & \\
\hline \multirow[t]{2}{*}{$a c r B$} & acrB-f & 531348 & TCGTGTTCCTGGTGATGTACCT & 68 & 66 & Baucheron et al., 2012 \\
\hline & acrB-r & 531281 & AACCGCAATAGTCGGAATCAA & & & \\
\hline \multirow[t]{2}{*}{$a c r F$} & acrF-f & 3563042 & GCTCTGTCGTCCATCTCAAAGA & 70 & 66 & This study \\
\hline & acrF-r & 3563111 & CGCGCTACAACGTTATAGTTTTCA & & & \\
\hline \multirow[t]{2}{*}{ tolC } & tolC-f & 3349107 & GCCCGTGCGCAATATGAT & 67 & 60 & Baucheron et al., 2012 \\
\hline & tolC-r & 3349173 & CCGCGTTATCCAGGTTGTTG & & & \\
\hline
\end{tabular}

${ }^{*}$ GenBank NC_003197.1.

${ }^{*} N A$ : Not Applicable due to the number of copies of this gene in Salmonella.

***2874 bp for S. Typhimurium or S. Paratyphi A and 539 bp for S. Typhi (see Figure 1). 
Transcription levels of efflux related genes acrA, acr $B, a c r F, t o l C$, $\operatorname{ram} A$, and $\operatorname{ramR}$ were done by quantitative reverse transcriptionPCR (qRT-PCR) as described previously (Baucheron et al., 2012; Giraud et al., 2013). Primers used for qRT-PCR are listed in (Table 2).

\section{RESULTS AND DISCUSSION RESISTANCE PHENOTYPES AND TARGET-AFFECTING MECHANISMS}

The twenty one $S$. Typhi and S. Paratyphi A strains of this study were isolated in France but acquired abroad, mainly in Asia and Africa (Table 1). Among the S. Typhi strains, all but two were $\mathrm{NAL}^{\mathrm{R}}(\mathrm{MIC}>16 \mathrm{mg} / \mathrm{L})$. One of the two strains was pansusceptible and the second was CIP ${ }^{\mathrm{DS}}$ but only categorized as intermediate for NAL (NAL $\left.{ }^{\mathrm{I}}, \mathrm{MIC} 16 \mathrm{mg} / \mathrm{L}\right)$. Of the $14 \mathrm{NAL}^{\mathrm{R}}$ strains, one was $\mathrm{CIP}^{R}, 12$ were $\mathrm{CIP}^{\mathrm{DS}}$ and one was $\mathrm{CIP}^{\mathrm{S}}$ (MIC $0.03 \mathrm{mg} / \mathrm{L})$. Eight $\mathrm{NAL}^{\mathrm{R}} S$. Typhi strains were also multidrug resistant. The majority of the $\mathrm{NAL}^{\mathrm{R}} S$. Typhi strains belonged to haplotype $\mathrm{H} 58$ which had emerged in Southern Asia during the mid 1990s (Roumagnac et al., 2006; Le et al., 2007). Among the $S$. Paratyphi A strains, all but two were $\mathrm{NAL}^{\mathrm{R}}$. One of the two strains was pansusceptible and the second was NAL ${ }^{\mathrm{I}}-\mathrm{CIP}^{\mathrm{DS}}$. The three others were NAL ${ }^{\mathrm{R}}$ - CIP ${ }^{\mathrm{DS}}$.

As shown in Table 1, both NAL ${ }^{\mathrm{I}}$-CIP ${ }^{\mathrm{DS}}$ had a mutation resulting in an amino acid substitution at position 464 of GyrB: serine to tyrosine for the $S$. Typhi isolate and serine to phenylalanine for the $S$. Paratyphi A isolate. The most frequent mechanism of resistance of $\mathrm{NAL}^{\mathrm{R}}-\mathrm{CIP}^{\mathrm{DS}}(n=17)$ and $\mathrm{NAL}^{\mathrm{R}}-\mathrm{CIP}^{S}(n=1)$ strains, whatever the serovar, was a substitution at position 83 (serine to phenylalanine, $n=12,66.6 \%$ ) of GyrA. Other GyrA modifications were observed at position 83 (serine to tyrosine) in two isolates or at position 87 (aspartic acid to glycine, aspartic acid to asparagine) in one isolate for each. As described previously, a single substitution in GyrA was associated with resistance to nalidixic acid and decreased susceptibility to CIP (Bhan et al., 2005; Le et al., 2007; Gaborieau et al., 2010; Hassing et al., 2011). One exception was the S. Typhi strain 4 (02) MB, which was $\mathrm{NAL}^{\mathrm{R}}-\mathrm{CIP}^{S}$ (and not $\mathrm{CIP}^{\mathrm{DS}}$ ) despite a mutation in gyrA resulting in substitution serine to tyrosine at position 83.

Additional substitutions were found in ParE of $2 \mathrm{NAL}^{\mathrm{R}}-\mathrm{CIP}^{\mathrm{DS}}$ $S$. Typhi strains that led to amino acid substitution aspartic acid to asparagine at position 420. In both cases, a 2-fold increase of NAL MICs was observed.

In the $\operatorname{CIP}^{\mathrm{R}} S$. Typhi isolate, three mutations leading to a double substitution in GyrA at positions 83 (serine to phenylalanine) and 87 (aspartic acid to asparagine) and one substitution at the position 80 of ParC (serine to isoleucine), as observed in previous studies (Renuka et al., 2005; Gaind et al., 2006; Lee et al., 2013).

The $\mathrm{NAL}^{\mathrm{R}}$ and CIP ${ }^{\mathrm{DS}} S$. Typhi and S. Paratyphi A strains harboring a single substitution in GyrA showed various values for NAL (64-1024 mg/L) and CIP (0.03-0.5 mg/L) MICs which suggested the presence of other mechanisms of resistance. Since the plasmid-mediated quinolone resistance-conferring genes $q n r A$, $q n r B$, qnrD or $q n r S$, qepA, and $a a c\left(6^{\prime}\right)-I b-c r$ were not detected, we investigated the role of the AcrAB-TolC efflux system.

\section{INVOLVEMENT OF EFFLUX}

None of the S. Typhi strains showed significant AcrA overproduction by dot blot, but nevertheless all $S$. Paratyphi A isolates showed a 2 or 3 -fold increased AcrA production relative to the susceptible $S$. Typhi isolate (Table $\mathbf{1}$ ). Thus, overproduction of AcrA seems not to be involved in CIP ${ }^{\mathrm{DS}}$ isolates compared with the susceptible isolates of $S$. Typhi or $S$. Paratyphi A. In presence of the efflux pump inhibitor PA $\beta \mathrm{N}$ (20 or $40 \mathrm{mg} / \mathrm{L}$ ), the CIP MICs similarly decreased ( 4 or 8 -fold) in CIP ${ }^{D S}$ and in susceptible strains (Table 3 and data not shown), which is in accordance with previous studies on $S$. Typhimurium and corresponds to

Table 3 | Study of efflux in a subset of Salmonella enterica serovars Typhi and Paratyphi A strains.

\begin{tabular}{|c|c|c|c|c|c|c|c|c|c|c|c|c|c|c|}
\hline Strain & $\begin{array}{l}\text { Antimicrobial } \\
\text { resistance pattern }\end{array}$ & \multicolumn{2}{|c|}{$\operatorname{MIC}(\mathrm{mg} / \mathrm{L})^{\mathrm{b}}$} & \multicolumn{4}{|c|}{ Substitution(s) in the QRDR of ${ }^{c}$ : } & $\begin{array}{l}\text { acrSE } \\
\text { sequencing }\end{array}$ & \multicolumn{6}{|c|}{ Transcription level of: } \\
\hline \multicolumn{15}{|c|}{ SALMONELLA TYPHI } \\
\hline $06-423$ & Pansusceptible & 4 & $0.015[0.004]$ & WT & WT & WT & WT & Deletion $^{d}$ & 1.0 & 1.0 & 1.0 & 1.0 & 1.0 & 1.0 \\
\hline 02-1180 & NALCIPDS & 64 & $0.125[0.015]$ & $\mathrm{D} 87 \mathrm{G}$ & WT & WT & WT & Deletiond & 1.5 & 0.7 & 0.5 & 0.8 & 0.9 & 1.4 \\
\hline 05-3275 & NALCIPDS & 64 & $0.125[0.030]$ & $\mathrm{D} 87 \mathrm{~N}$ & WT & WT & WT & Deletion ${ }^{d}$ & 0.5 & 1.4 & 0.2 & 0.5 & 0.3 & 0.7 \\
\hline \multicolumn{15}{|c|}{ SALMONELLA PARATYPHI A } \\
\hline 08-8903 & Pansusceptible & 8 & $0.030[0.008]$ & WT & WT & WT & WT & WT & 1.0 & 1.0 & 1.0 & 1.0 & 1.0 & 1.0 \\
\hline 07-6329 & CIPDS & 16 & $0.25[0.060]$ & WT & S464F & WT & WT & WT & 1.6 & 1.4 & 1.0 & 1.0 & 1.0 & 1.2 \\
\hline
\end{tabular}

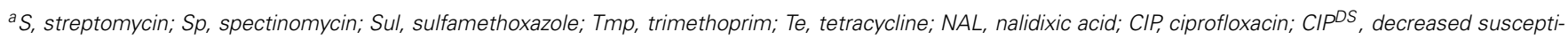
bility to ciprofloxacin.

${ }^{b}$ Values in brackets are MICs in the presence of the efflux pump inhibitor PA $\beta N$ at $40 \mathrm{mg} / \mathrm{L}$.

' WT, wild type; $D$, aspartic acid; F, phenylalanine; G, glycine; I, isoleucine; $N$, asparagine; $S$, serine.

d 2334 bp deleted. 


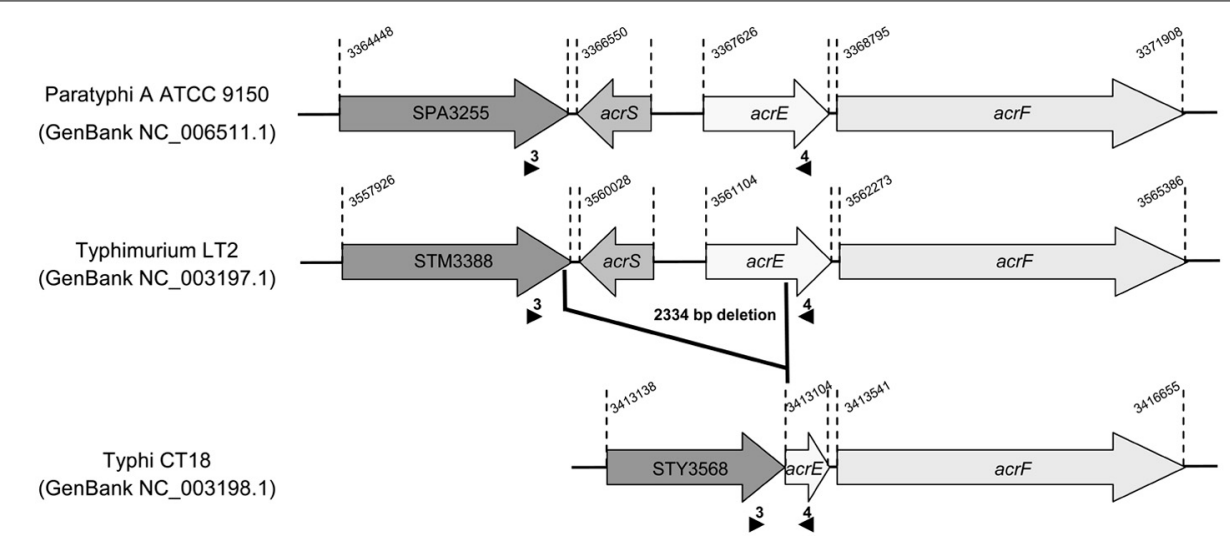

FIGURE 1 | Deletion identified in the acrSE region relative to the genome sequence of $S$. Typhi strain CT18 (GenBank NC_003198.1). The sequence of the acrSE region of $S$. Typhi strain CT18 is compared to those of S. Paratyphi A strain ATCC9150 (GenBank NC_006511.1) and
S. Typhi strain LT2 (GenBank NC_003197.1). Small black arrows indicate primers positions used for PCR to amplify and sequence the acrSE region. The $2334 \mathrm{bp}$ chromosomal deletion was found in all $S$. Typhi strains studied. a decrease of resistance level observed for $a c r B$ or tolC deletion mutants (Baucheron et al., 2002, 2004b).

Despite a lack of evidence of increased efflux in the resistance phenotype, we measured by qRT-PCR the transcription levels of efflux related genes $a c r A, a c r F, a c r B, t o l C, \operatorname{ram} A$, and ramR in $\mathrm{CIP}^{\mathrm{DS}}$ non-MDR strains and in the $\mathrm{CIP}^{\mathrm{R}}$ strain. No differences were detected in the transcription levels of these genes, between susceptible, CIP DS and $\mathrm{CIP}^{\mathrm{R}}$ strains, whatever the serovar (Table 3 ). In addition, no mutations were detected in the regulatory regions of the AcrAB-TolC efflux system. However, during the screening of the regulatory regions, we identified a single large deletion of 2334 bp in the acrS-acrE region of all $S$. Typhi strains, including the susceptible one (Table 3). This deletion encompassed the acrS gene, that encodes a transcriptional repressor, and a large part of the acrE gene that encodes the AcrE periplasmic lipoprotein, which is homologous to AcrA (Olliver et al., 2005). This 2334 bp deletion was also observed in the acrS-acrE region of sequenced genomes of MDR S. Typhi CT18 strain (Parkhill et al., 2001) and pansusceptible TY2 strain (Deng et al., 2003) (Figure 1). Previously, it has been shown that acrS deletion in $S$. Typhimurium does not affect acrEF expression (Olliver et al., 2005). Similarly the natural acrSE deletion detected in $S$. Typhi had no impact on the acrF transcription level as observed in this study. To our knowledge, this is the first description of such a natural acrS-acrE chromosomal deletion and seems specific to $S$. Typhi since it was not detected in all currently sequenced genomes of the other serovars (not shown).

\section{CONCLUSIONS}

The main mechanisms involved in quinolone or FQ resistance in both S. Typhi and S. Paratyphi A are target modifications. In contrast to what is seen in enteric pathogenic serovars, such as Typhimurium or the emerging CIP ${ }^{\mathrm{R}}$ Kentucky ST198 clone (Baucheron et al., 2013), increased efflux pump productionmediated mechanisms seem to be totally absent in both $S$. Typhi and $S$. Paratyphi A. The deletion identified in the acrSEF region, although not involved in the resistance phenotype, may be helpful for the specific detection of $S$. Typhi.

\section{ACKNOWLEDGMENTS}

We are grateful to Dr. Hong Le for providing some of the studied strains and to Laëtitia Fabre for excellent technical assistance. We would like to thank all corresponding laboratories of the French National Reference Center for E. coli, Shigella, and Salmonella. The French National Reference Center for E. coli, Shigella, and Salmonella is funded by the Institut Pasteur and the Institut de Veille Sanitaire. The "Unité des Bactéries Pathogènes Entériques" belongs to the "Integrative Biology of Emerging Infectious Diseases" Laboratory of Excellence funded by the French Government "Investissement d'Avenir" programme (grant no. ANR-10-LABX-62-IBEID).

\section{REFERENCES}

Abouzeed, Y. M., Baucheron, S., and Cloeckaert, A. (2008). ramR mutations involved in efflux-mediated multidrug resistance in Salmonella enterica serovar Typhimurium. Antimicrob. Agents Chemother. 52, 2428-2434. doi: 10.1128/ AAC.00084-08

Accou-Demartin, M., Gaborieau, V., Song, Y., Roumagnac, P., Marchou, B., Achtman, M., et al. (2011). Salmonella enterica Serotype Typhi with nonclassical quinolone resistance phenotype. Emerg. Infect. Dis. 17, 1091-1094. doi: 10.3201/ eid/1706.101242

Akiyama, T., and Khan, A. A. (2012). Molecular characterization of strains of fluoroquinolone-resistant Salmonella enterica serovar Schwarzengrund carrying multidrug resistance isolated from imported foods. J. Antimicrob. Chemother. 67, 101-110. doi: 10.1093/jac/dkr414

Bailey, A. M., Ivens, A., Kingsley, R., Cottell, J. L., Wain, J., and Piddock, L. J. V. (2010). RamA, a member of the AraC/XylS family, influences both virulence and efflux in Salmonella enterica serovar Typhimurium. J. Bacteriol. 192, 1607-1616. doi: 10.1128/JB.01517-09

Baucheron, S., Chaslus-Dancla, E., and Cloeckaert, A. (2004a). Role of TolC and parC mutation in high-level fluoroquinolone resistance in Salmonella enterica serotype Typhimurium DT204. J. Antimicrob. Chemother. 53, 657-659. doi: 10.1093/jac/dkh122

Baucheron, S., Tyler, S., Boyd, D., Mulvey, M. R., Chaslus-Dancla, E., and Cloeckaert A. (2004b). AcrAB-TolC directs efflux-mediated multidrug resistance in Salmonella enterica serovar Typhimurium DT104. Antimicrob. Agents Chemother. 48, 3729-3735. doi: 10.1128/AAC.48.10.3729-3735.2004

Baucheron, S., Coste, F., Canepa, S., Maurel, M. C., Giraud, E., Culard, F., et al. (2012). Binding of the RamR repressor to wild-type and mutated promoters of the RamA gene involved in efflux-mediated multidrug resistance in Salmonella enterica serovar Typhimurium. Antimicrob. Agents Chemother. 56, 942-948. doi: 10.1128/AAC.05444-11 
Baucheron, S., Imberechts, H., Chaslus-Dancla, E., and Cloeckaert, A. (2002). The AcrB multidrug transporter plays a major role in high-level fluoroquinolone resistance in Salmonella enterica serovar Typhimurium phage type DT204. Microb. Drug Resist. 8, 281-289. doi: 10.1089/10766290260469543

Baucheron, S., Le Hello, S., Doublet, B., Giraud, E., Weill, F. X., and Cloeckaert, A. (2013). $\operatorname{ram} R$ mutations affecting fluoroquinolone susceptibility in epidemic multidrug-resistant Salmonella enterica serovar Kentucky ST198. Front. Microbiol. 4:213. doi: 10.3389/fmicb.2013.00213

Bhan, M. K., Bahl, R., and Bhatnagar, S. (2005). Typhoid and paratyphoid fever. Lancet 366, 749-762. doi: 10.1016/S0140-6736(05)67181-4

Cloeckaert, A., and Chaslus-Dancla, E. (2001). Mechanisms of quinolone resistance in Salmonella. Vet. Res. 32, 291-300. doi: 10.1051/vetres:2001105

Connor, B. A., and Schwartz, E. (2005). Typhoid and paratyphoid fever in travellers. Lancet Infect. Dis. 5, 623-628. doi: 10.1016/S1473-3099(05)70239-5

Crump, J. A., Luby, S. P., and Mintz, E. D. (2004). The global burden of typhoid fever. Bull. World Health Organ. 82, 346-353.

Crump, J. A., and Mintz, E. D. (2010). Global trends in typhoid and paratyphoid Fever. Clin. Infect. Dis. 50, 241-246. doi: 10.1086/649541

Deng, W., Liou, S. R., Plunkett, G. 3rd., Mayhew, G. F., Rose, D. J., Burland, V., et al. (2003). Comparative genomics of Salmonella enterica serovar Typhi strains Ty2 and CT18. J. Bacteriol. 185, 2330-2337. doi: 10.1128/JB.185.7.2330-2337.2003

Gaborieau, V., Weill, F. X., and Marchou, B. (2010). A case of Salmonella enterica serovar Typhi with decreased susceptibility to ciprofloxacin. Med. Mal. Infect. 40, 691-695. doi: 10.1016/j.medmal.2010.06.009

Gaind, R., Paglietti, B., Murgia, M., Dawar, R., Uzzau, S., Cappuccinelli, P., et al. (2006). Molecular characterization of ciprofloxacin-resistant Salmonella enterica serovar Typhi and Paratyphi A causing enteric fever in India. J. Antimicrob. Chemother. 58, 1139-1144. doi: 10.1093/jac/dkl391

Giraud, E., Baucheron, S., and Cloeckaert, A. (2006). Resistance to fluoroquinolones in Salmonella: emerging mechanisms and resistance prevention strategies. Microbes Infect. 8, 1937-1944. doi: 10.1016/j.micinf.2005.12.025

Giraud, E., Baucheron, S., Virlogeux-Payant, I., Nishino, K., and Cloeckaert, A. (2013). Effects of natural mutations in the ramRA locus on invasiveness of epidemic fluoroquinolone-resistant Salmonella enterica serovar Typhimurium isolates. J. Infect. Dis. 207, 794-802. doi: 10.1093/infdis/jis755

Giraud, E., Cloeckaert, A., Kerboeuf, D., and Chaslus-Dancla, E. (2000). Evidence for active efflux as the primary mechanism of resistance to ciprofloxacin in Salmonella enterica serovar Typhimurium. Antimicrob. Agents Chemother. 44, 1223-1228. doi: 10.1128/AAC.44.5.1223-1228.2000

Hassing, R. J., Goessens, W. H., Mevius, D. J., van Pelt, W., Mouton, J. W., Verbon, A., et al. (2013). Decreased ciprofloxacin susceptibility in Salmonella Typhi and Paratyphi infections in ill-returned travellers: the impact on clinical outcome and future treatment options. Eur. J. Clin. Microbiol. Infect. Dis. 32, 1295-1301. doi: 10.1007/s10096-013-1878-9

Hassing, R. J., Menezes, G. A., van Pelt, W., Petit, P. L., van Genderen, P. J., and Goessens, W. H. (2011). Analysis of mechanisms involved in reduced susceptibility to ciprofloxacin in Salmonella enterica serotypes Typhi and Paratyphi A isolates from travellers to Southeast Asia. Int. J. Antimicrob. Agents 37, 240-243. doi: 10.1016/j.ijantimicag.2010.10.026

Hentschke, M., Christner, M., Sobottka, I., Aepfelbacher, M., and Rohde, H. (2010). Combined ramR mutation and presence of a Tn1721-associated tet(A) variant in a clinical isolate of Salmonella enterica serovar Hadar resistant to tigecycline. Antimicrob. Agents Chemother. 54, 1319-1322. doi: 10.1128/AAC.00993-09

Kehrenberg, C., Cloeckaert, A., Klein, G., and Schwarz, S. (2009). Decreased fluoroquinolone susceptibility in mutants of Salmonella serovars other than Typhimurium: detection of novel mutations involved in modulated expression of ramA and soxS. J. Antimicrob. Chemother. 64, 1175-1180. doi: 10.1093/jac/ dkp347

Le, T. A., Fabre, L., Roumagnac, P., Grimont, P. A., Scavizzi, M. R., and Weill, F. X. (2007). Clonal expansion and microevolution of quinolone-resistant Salmonella enterica serotype Typhi in Vietnam from 1996 to 2004. J. Clin. Microbiol. 45, 3485-3492. doi: 10.1128/JCM.00948-07

Lee, C. J., Su, L. H., Huang, Y. C., and Chiu, C. H. (2013). First isolation of ciprofloxacin-resistant Salmonella enterica serovar Typhi in Taiwan. J. Microbiol. Immunol. Infect. 46, 469-473. doi: 10.1016/j.jmii.2013.01.002

Nikaido, E., Yamaguchi, A., and Nishino, K. (2008). AcrAB multidrug efflux pump regulation in Salmonella enterica serovar Typhimurium by RamA in response to environmental signals. J. Biol. Chem. 283, 24245-24253. doi: 10.1074/jbc. M804544200

Ochiai, R. L., Wang, X., von Seidlein, L., Yang, J., Bhutta, Z. A., Bhattacharya, S. K., et al. (2005). Salmonella paratyphi A rates, Asia. Emerg. Infect. Dis. 11, 1764-1766. doi: 10.3201/eid1111.050168

Olliver, A., Valle, M., Chaslus-Dancla, E., and Cloeckaert, A. (2005). Overexpression of the multidrug efflux operon acrEF by insertional activation with IS1 or IS10 elements in Salmonella enterica serovar typhimurium DT204 acrB mutants selected with fluoroquinolones. Antimicrob. Agents Chemother. 49, 289-301. doi: 10.1128/AAC.49.1.289-301.2005

Parkhill, J., Dougan, G., James, K. D., Thomson, N. R., Pickard, D., Wain, J., et al. (2001). Complete genome sequence of a multiple drug resistant Salmonella enterica serovar Typhi CT18. Nature 413, 848-852. doi: 10.1038/35101607

Piddock, L. J. V. (2002). Fluoroquinolone resistance in Salmonella serovars isolated from humans and food animals. FEMS Microbiol. Rev. 26, 3-16. doi: 10.1016/ S0168-6445(01)00076-6

Piddock, L. J. V. (2006). Clinically relevant chromosomally encoded multidrug resistance efflux pumps in bacteria. Clin. Microbiol. Rev. 19, 382-402. doi: 10.1128/CMR.19.2.382-402.2006

Renuka, K., Sood, S., Das, B. K., and Kapil, A. (2005). High-level ciprofloxacin resistance in Salmonella enterica serotype Typhi in India. J. Med. Microbiol. 54, 999-1000. doi: 10.1099/jmm.0.45966-0

Roumagnac, P., Weill, F. X., Dolecek, C., Baker, S., Brisse, S., Chinh, N. T., et al. (2006). Evolutionary history of Salmonella Typhi. Science 314, 1301-1304. doi: $10.1126 /$ science. 1134933

Song, Y., Roumagnac, P., Weill, F. X., Wain, J., Dolecek, C., Mazzoni, C. J., et al. (2010). A multiplex single nucleotide polymorphism typing assay for detecting mutations that result in decreased fluoroquinolone susceptibility in Salmonella enterica serovars Typhi and Paratyphi A. J. Antimicrob. Chemother. 65, 1631-1641. doi: 10.1093/jac/dkq175

Velge, P., Cloeckaert, A., and Barrow, P. (2005). Emergence of Salmonella epidemics: the problems related to Salmonella enterica serotype Enteritidis and multiple antibiotic resistance in other major serotypes. Vet. Res. 36, 267-288. doi: 10.1051/vetres:2005005

Conflict of Interest Statement: The authors declare that the research was conducted in the absence of any commercial or financial relationships that could be construed as a potential conflict of interest.

Received: 30 October 2013; accepted: 09 January 2014; published online: 27 January 2014.

Citation: Baucheron S, Monchaux I, Le Hello S, Weill F-X and Cloeckaert A (2014) Lack of efflux mediated quinolone resistance in Salmonella enterica serovars Typhi and Paratyphi A. Front. Microbiol. 5:12. doi: 10.3389/fmicb.2014.00012

This article was submitted to Antimicrobials, Resistance and Chemotherapy, a section of the journal Frontiers in Microbiology.

Copyright (C) 2014 Baucheron, Monchaux, Le Hello, Weill and Cloeckaert. This is an open-access article distributed under the terms of the Creative Commons Attribution License (CC BY). The use, distribution or reproduction in other forums is permitted, provided the original author(s) or licensor are credited and that the original publication in this journal is cited, in accordance with accepted academic practice. No use, distribution or reproduction is permitted which does not comply with these terms. 